\title{
ON THE ERROR TERM OF THE LOGARITHM OF THE LCM OF A QUADRATIC SEQUENCE
}

\author{
JUANJO RUÉ, PAULIUS ŠARKA, AND ANA ZUMALACÁRREGUI
}

\begin{abstract}
We study the logarithm of the least common multiple of the sequence of integers given by $1^{2}+1,2^{2}+1, \ldots, n^{2}+1$. Using a result of Homma 4 on the distribution of roots of quadratic polynomials modulo primes we calculate the error term for the asymptotics obtained by Cilleruelo [2].
\end{abstract}

\section{INTRODUCTION}

The first important attempt to prove the Prime Number Theorem was made by Chebyshev. In 1853 [2] he introduced the function $\psi(n)=\sum_{p^{m}<n} \log (p)$ and proved that the Prime Number Theorem was equivalent to the asymptotic estimate $\psi(n) \sim n$. He also proved that if $\psi(n) / n$ had a limit as $n$ tends to infinity then that limit is 1 . The proof of this result was only completed (independently) two years after Chebyshev's death by Hadamard and de la Vallée Poussin.

Observe that Chebyshev's function is precisely $\psi(n)=\log \operatorname{lcm}(1,2, \ldots, n)$, so it seems natural to consider the following question: for a given polynomial $f(x) \in \mathbb{Z}[x]$, what can be said about the $\log \operatorname{lcm}(f(1), f(2), \ldots, f(n))$ ? As Hadamard and de la Vallée Poussin proved, for $f(x)=x$ this quantity asymptotically behaves as $n$. Some progress has been made in the direction of generalising this result to a broader class of polynomials. In [1] the authors use the Prime Number Theorem for arithmetic progressions to get the asymptotic estimate for any linear polynomial $f(x)=a x+b$ :

$$
\log \operatorname{lcm}(f(1), f(2), \ldots, f(n)) \sim n \frac{q}{\varphi(q)} \sum_{\substack{k=1 \\(k, q)=1}}^{q} \frac{1}{k},
$$

where $q=a / \operatorname{gcd}(a, b)$. Recently, Cilleruelo 2 extended this result to the quadratic case, obtaining that for an irreducible polynomial $f(x)=a x^{2}+b x+c \in \mathbb{Z}[x]$ the following asymptotic estimate holds:

$$
\log \operatorname{lcm}(f(1), f(2), \ldots, f(n))=n \log n+B_{f} n+o(n),
$$

where the constant $B_{f}$ is explicit. The author also proves that for reducible polynomials of degree two, the asymptotic is linear in $n$. For polynomials of higher degree nothing is known, except for products of linear polynomials, which are studied in $[5]$.

An important ingredient in Cilleruelo's argument is a result of Toth 9, a generalisation of a deep theorem of Duke, Friedlander and Iwaniec 3 about the distribution of solutions of quadratic congruences $f(x) \equiv 0(\bmod p)$, when $p$ runs over all primes. Recent improvements of the latter result in the negative discriminant case [4] allowed us to refine the method and obtain the error term in the special case of expression (11).

We focus our study on the particular polynomial $f(x)=x^{2}+1$, which simplifies the calculation, and shows how the method developed in [2] works in a clear manner. The same ideas could be extended to general irreducible quadratic polynomials of negative discriminant, however, a generalisation of [4] (in the same direction as Toth's) would be necessary.

For this particular polynomial the expression for $B$ is given by

$$
\gamma-1-\frac{\log 2}{2}-\sum_{p \neq 2} \frac{\left(\frac{-1}{p}\right) \log p}{p-1} \approx-0.0662756342,
$$


where $\gamma$ is the Euler constant, $\left(\frac{-1}{p}\right)$ is the Legendre symbol and the sum is considered over all odd prime numbers ( $B$ can be computed with high numerical precision by using its expression in terms of L-series and zeta-series, see [2] for details). More precisely, we obtain the following estimate:

Theorem 1.1. For any $\theta<4 / 9$ we have

$$
\log \operatorname{lcm}\left(1^{2}+1,2^{2}+1, \ldots, n^{2}+1\right)=n \log n+B n+O\left(\frac{n}{(\log n)^{\theta}}\right),
$$

where the constant B is given by Expression (2).

The infinite sum in (2) appears in other mathematical contexts: as it is pointed in [7] this sum is closely related to multiplicative sets whose elements are non-hypotenuse numbers (i.e. integers which could not be written as the hypothenuse of a right triangle with integer sides).

Plan of the paper: in Section 2 we recall the basic necessary results and fix the notation used in the rest of the paper. We explain the strategy for the proof of Theorem 1.1 in Section 3 , which is based on a detailed study of medium primes (Section 44). Then, using these partial results, in Section 5 we provide the complete proof of Theorem 1.1

\section{BACKGROUND AND NOTATION}

Throughout the paper $p$ will denote a prime number and the Landau symbols $O$ and $o$, as well as the Vinogradov symbols $\ll$, 》 will be employed with their usual meaning. We will also use the following notation:

$$
\begin{aligned}
\pi(n) & =|\{p: p \leq n\}|, \\
\pi_{1}(n) & =|\{p: p \equiv 1(\bmod 4), p \leq n\}|, \\
\pi_{1}([a, b]) & =|\{p: p \equiv 1(\bmod 4), a<p \leq b\}| .
\end{aligned}
$$

The Prime Number Theorem states that the following estimate holds:

$$
\psi(n)=\log \operatorname{lcm}(1,2, \ldots, n)=n+E(n), E(n)=O\left(\frac{n}{(\log n)^{\kappa}}\right),
$$

where $\kappa$ can be chosen as large as necessary. We also use the following estimate, which follows from Prime Number Theorem for arithmetic progressions:

$$
\pi_{1}(n)=\frac{n}{2 \log n}+O\left(\frac{n}{(\log n)^{2}}\right) .
$$

The result needed in order to refine the error term of (1) is the main theorem in [4, which deals with the distribution of fractional parts $\nu / p$, where $p$ is a prime less than or equal to $n$ and $\nu$ is a root in $\mathbb{Z} / p \mathbb{Z}$ of a quadratic polynomial $f(x)$ with negative discriminant. For this $f$, we define the discrepancy $D_{f}(n)$ associated to the set of fractions $\{\nu / p: f(\nu) \equiv 0(\bmod p), p \leq n\}$ as

$$
D_{f}(n)=\sup _{[u, v] \in[0,1]}||[u, v]\left|-\frac{1}{\pi(n)} \sum_{p \leq n} \sum_{\substack{u<\nu / p \leq v \\ f(\nu) \equiv 0(\bmod p)}} 1\right|,
$$

where $|[u, v]|:=v-u$. Under these assumptions, the main theorem of [4] can be stated as follows:

Theorem 2.1. Let $f$ be any irreducible quadratic polynomial with integer coefficients and negative discriminant. Then for any $\delta<8 / 9$ we have

$$
D_{f}(n)=O\left(\frac{1}{(\log n)^{\delta}}\right) .
$$

As a consequence of this result, we have the following lemma: 
Lemma 2.1. Let $g:[0,1] \rightarrow \mathbb{R}$ be any function of bounded variation, and $n<N$ two positive real numbers. Then for any $\delta<8 / 9$

$$
\sum_{\substack{n<p<N \\ 0 \leq \nu<p \\ \nu^{2} \equiv-1(\bmod p)}} g\left(\frac{\nu}{p}\right)=2 \pi_{1}([n, N]) \int_{0}^{1} g(t) \mathrm{d} t+O\left(\frac{N}{(\log N)^{1+\delta}}\right) .
$$

Proof. We know by the Koksma-Hlawka identity (see Theorem 2.11 in [8]) that for any sequence $A=\left\{a_{1}, a_{2}, \ldots, a_{n}\right\}, A \subset[0,1]$, with discrepancy $D(n)$ and for any $g:[0,1] \rightarrow \mathbb{R}$ with bounded variation, we have

$$
\frac{1}{n} \sum_{i=1}^{n} g\left(a_{i}\right)=\int_{0}^{1} g(t) \mathrm{d} t+O(D(n))
$$

so

$$
\sum_{i=n}^{N} g\left(a_{i}\right)=\sum_{i=1}^{N} g\left(a_{i}\right)-\sum_{i=1}^{n} g\left(a_{i}\right)=(N-n) \int_{0}^{1} g(t) \mathrm{d} t+O(N D(N))+O(n D(n)) .
$$

In our case, using Theorem 2.1, we get

$$
\sum_{\substack{n<p<N \\ 0 \leq \nu<p \\ \equiv \leq-1(\bmod p)}} g\left(\frac{\nu}{p}\right)=2 \pi_{1}([n, N]) \int_{0}^{1} g(t) \mathrm{d} t+O\left(\frac{\pi_{1}(N)}{(\log N)^{\delta}}\right) .
$$

Using rough estimate $\pi_{1}(N)=O\left(\frac{N}{\log N}\right)$ we get the required error term.

\section{The STRATEgy}

The content of this section can be found in [2. We include it here for completeness and to prepare the reader for the forthcoming arguments.

Denote by $P_{n}=\prod_{i=1}^{n}\left(i^{2}+1\right)$ and $L_{n}=\operatorname{lcm}\left(1^{2}+1,2^{2}+1, \ldots, n^{2}+1\right)$, and write $\alpha_{p}(n)=$ $\operatorname{ord}_{p}\left(P_{n}\right)$ and $\beta_{p}(n)=\operatorname{ord}_{p}\left(L_{n}\right)$. The argument for estimating $L_{n}$ stems from the following equality:

$$
\log L_{n}=\log P_{n}+\sum_{p}\left(\beta_{p}(n)-\alpha_{p}(n)\right) \log p .
$$

Clearly it is not difficult to estimate $\log P_{n}$. Indeed, using Stirling's approximation formula, we get

$$
\log \prod_{i=i}^{n}\left(i^{2}+1\right)=\log \prod_{i=1}^{n} i^{2}+\log \prod_{i=1}^{n}\left(1+\frac{1}{i^{2}}\right)=2 \log n !+O(1)=2 n \log n-2 n+O(\log n),
$$

and so in the remainder of the paper we will be concerned with the estimation of $\sum_{p}\left(\beta_{p}(n)-\right.$ $\left.\alpha_{p}(n)\right) \log p$. We start here by making three simple observations:

\section{Lemma 3.1.}

i) $\beta_{2}(n)-\alpha_{2}(n)=-n / 2+O(1)$
ii) $\beta_{p}(n)-\alpha_{p}(n)=0$, when $p>2 n$
iii) $\beta_{p}(n)=\alpha_{p}(n)=0$, when $p \equiv 3(\bmod 4)$

Proof.

i) $i^{2}+1$ is never divisible by 4 and is divisible by 2 for every odd $i$.

ii) Note that $\alpha_{p}(n) \neq \beta_{p}(n)$ only if there exist $i<j \leq n$ such that $p \mid i^{2}+1$ and $p \mid j^{2}+1$. But this implies $p \mid(i-j)(i+j)$, and in turn $p \leq 2 n$.

iii) $i^{2}+1$ is never divisible by $p \equiv 3(\bmod 4)$ as -1 is not a quadratic residue modulo such prime. 
Since we have dealt with the prime 2, from now on we will only consider odd primes. Lemma 3.1 also states that it is sufficient to study the order of prime numbers which are smaller than $2 n$ and are equivalent to 1 modulo 4 . We split these primes in two groups: ones that are smaller than $n^{2 / 3}$ and others that are between $n^{2 / 3}$ and $2 n$, small and medium primes respectively.

The computation for small primes is easy and it is done in the lemma below, after obtaining simple estimates for $\alpha_{p}(n)$ and $\beta_{p}(n)$. Analysis of medium primes, which is left for the next section, is more subtle and will lead to improvement of the error term.

Lemma 3.2. For primes $p \equiv 1(\bmod 4)$ the following estimates hold:

i) $\beta_{p}(n) \ll \frac{\log n}{\log p}$,

ii) $\alpha_{p}(n)=\frac{2 n}{p-1}+O\left(\frac{\log n}{\log p}\right)$.

Proof.

i) It is clear that $\beta_{p}(n)$ satisfies $p^{\beta_{p}(n)} \leq n^{2}+1$, so

$$
\beta_{p}(n) \leq \frac{\log \left(n^{2}+1\right)}{\log p} \ll \frac{\log n}{\log p} .
$$

ii) In order to estimate $\alpha_{p}(n)$ note that for primes $p \equiv 1(\bmod 4)$ equation $i^{2} \equiv-1\left(\bmod p^{a}\right)$ has two solutions $\nu_{1}$ and $\nu_{2}$ in the interval $\left[1, p^{a}\right]$ and every other solution is of the form $\nu_{1}+k p^{a}$ or $\nu_{2}+k p^{a}, k \in \mathbb{Z}$. The number of times $p^{a}$ divides $i^{2}+1, i=1, \ldots, n$ is given by

$$
2+\left\lfloor\frac{n-\nu_{1}}{p^{a}}\right\rfloor+\left\lfloor\frac{n-\nu_{2}}{p^{a}}\right\rfloor,
$$

which equals to 0 for $p^{a}>n^{2}+1$ and $2 n / p^{a}+O(1)$ for $p^{a} \leq n^{2}+1$. Therefore we get

$$
\begin{aligned}
\alpha_{p}(n) & =2 \sum_{j=1}^{\left\lfloor\frac{\log \left(n^{2}+1\right)}{\log p}\right\rfloor} \frac{n}{p^{j}}+O\left(\frac{\log n}{\log p}\right) \\
& =2 n \sum_{j=1}^{\infty} \frac{1}{p^{j}}-2 n \sum_{j=\left\lfloor\frac{\log \left(n^{2}+1\right)}{\log p}\right\rfloor+1}^{\infty} \frac{1}{p^{j}}+O\left(\frac{\log n}{\log p}\right) \\
& =\frac{2 n}{p-1}+O\left(\frac{\log n}{\log p}\right),
\end{aligned}
$$

and the claim follows.

Lemma 3.3. The following estimate holds:

$$
\sum_{2<p<n^{2 / 3}}\left(\alpha_{p}(n)-\beta_{p}(n)\right) \log p=\sum_{2<p<n^{2 / 3}} \frac{\left(1+\left(\frac{-1}{p}\right)\right) n \log p}{p-1}+O\left(n^{2 / 3}\right) .
$$

Proof. Using the estimates from Lemma 3.2 we get

$$
\sum_{2<p<n^{2 / 3}} \beta_{p}(n) \log p \ll \sum_{2<p<n^{2 / 3}} \log n \ll n^{2 / 3},
$$

and also

$$
\begin{aligned}
\sum_{2<p<n^{2 / 3}} \alpha_{p}(n) \log p & =\sum_{\substack{p<n^{2 / 3} \\
p \equiv 1(\bmod 4)}}\left(\frac{2 n \log p}{p-1}+O(\log n)\right) \\
& =\sum_{2<p<n^{2 / 3}} \frac{\left(1+\left(\frac{-1}{p}\right)\right) n \log p}{p-1}+O\left(n^{2 / 3}\right),
\end{aligned}
$$

and hence the claim follows. 


\section{Medium PRImes}

In order to deal with the remaining primes, we note, that if prime $p \equiv 1(\bmod 4)$ is in the range $n^{2 / 3} \leq p \leq 2 n$ then it divides $i^{2}+1$ for some $i \leq n$. However, since such a prime is sufficiently large compared to $n^{2}+1$, the case that $p^{2}$ divides some $i^{2}+1, i \leq n$ is unlikely.

Having this in mind, we separate contribution of higher degrees from the contribution of degree 1 . Define for $p \equiv 1(\bmod 4)$ :

$$
\begin{aligned}
& \alpha_{p}^{*}(n)=\left|\left\{i: p \mid i^{2}+1, i \leq n\right\}\right|, \\
& \beta_{p}^{*}(n)=1,
\end{aligned}
$$

and, for $p \equiv 3(\bmod 4), \alpha_{p}^{*}(n)=\beta_{p}^{*}(n)=0$. Then

$$
\begin{aligned}
\sum_{n^{2 / 3} \leq p \leq 2 n}\left(\beta_{p}(n)-\alpha_{p}(n)\right) \log p= & \sum_{n^{2 / 3} \leq p \leq 2 n}\left(\beta_{p}(n)-\beta_{p}^{*}(n)-\alpha_{p}(n)+\alpha_{p}^{*}(n)\right) \log p+ \\
& \sum_{n^{2 / 3} \leq p \leq 2 n} \beta_{p}^{*}(n) \log p-\sum_{n^{2 / 3} \leq p \leq 2 n} \alpha_{p}^{*}(n) \log p .
\end{aligned}
$$

We now estimate each sum in the previous equation. We start estimating the first one:

Lemma 4.1. The following estimate holds:

$$
\sum_{n^{2 / 3} \leq p \leq 2 n}\left(\beta_{p}(n)-\beta_{p}^{*}(n)-\alpha_{p}(n)+\alpha_{p}^{*}(n)\right) \log p \ll n^{2 / 3} \log n .
$$

To prove this lemma we need some preliminary results. As it was intended, $\left(\beta_{p}(n)-\beta_{p}^{*}(n)-\right.$ $\left.\alpha_{p}(n)+\alpha_{p}^{*}(n)\right) \log p$ is nonzero only when $p^{2} \mid i^{2}+1$ for some $i \leq n$. We claim, that number of such primes is small:

Lemma 4.2. The following estimate holds:

$$
\left|\left\{p: p^{2} \mid i^{2}+1, n^{2 / 3} \leq p \leq 2 n, i \leq n\right\}\right| \ll n^{2 / 3} .
$$

Proof. Let us split the interval $\left[n^{2 / 3}, 2 n\right]$ into dyadic intervals, consider one of them, say $[Q, 2 Q]$, and define

$$
P_{k}=\left\{p: i^{2}+1=k p^{2} \text { for some } i \leq n\right\} .
$$

We estimate the size of the set $P_{k} \cap[Q, 2 Q]$, which is nonempty only when $k \leq\left(n^{2}+1\right) / Q^{2}$. For every $p \in P_{k} \cap[Q, 2 Q]$ we have $i^{2}-k p^{2}=(i+\sqrt{k} p)(i-\sqrt{k} p)=-1$, thus

$$
\left|\frac{i}{p}-\sqrt{k}\right|=\frac{1}{p^{2}}\left(\frac{i}{p}+\sqrt{k}\right)^{-1} \leq \frac{1}{p^{2}} \leq \frac{1}{Q^{2}} .
$$

On the other hand, all fractions $i / p, p \in P_{k}$, are pairwise different, since $i p^{\prime}=i^{\prime} p$ implies $p=p^{\prime}$ (otherwise $p \mid i$, and so $p \mid i^{2}-k p^{2}=-1$ ), therefore

$$
\left|\frac{i}{p}-\frac{i^{\prime}}{p^{\prime}}\right| \geq \frac{1}{p p^{\prime}} \gg \frac{1}{Q^{2}} .
$$

Combining both inequalities we get $\left|P_{k} \cap[Q, 2 Q]\right| \ll 1$ for every $k \leq\left(n^{2}+1\right) / Q^{2}$. Recalling that $P_{k} \cap[Q, 2 Q]$ is empty for other values of $k$ we have

$$
\left|\left\{p: p^{2} \mid i^{2}+1, Q \leq p \leq 2 Q, i \leq n\right\}\right|=\left|\cup_{k}\left(P_{k} \cap[Q, 2 Q]\right)\right| \ll \frac{n^{2}}{Q^{2}} .
$$

Summing over all dyadic intervals the result follows.

Now we use this estimate to prove Lemma 4.1 
Proof of Lemma 4.1. We use estimates from Lemma 3.2 and the estimate for $\alpha_{p}^{*}(n)$, which follows from Expression (5):

$$
\begin{aligned}
& \beta_{p}(n) \ll \frac{\log n}{\log p}, \\
& \alpha_{p}(n)=\frac{2 n}{p-1}+O\left(\frac{\log n}{\log p}\right), \\
& \alpha_{p}^{*}(n)=\frac{2 n}{p}+O(1) .
\end{aligned}
$$

For any prime $n^{2 / 3}<p<2 n$, such that $p^{2} \mid i^{2}+1$ for some $i \leq n$, we get

$$
\left|\beta_{p}(n)-\beta_{p}^{*}(n)-\alpha_{p}(n)+\alpha_{p}^{*}(n)\right|=\frac{2 n}{p(p-1)}+O\left(\frac{\log n}{\log p}\right) \ll \frac{\log n}{\log p} .
$$

It follows from lemma 4.2 that the number of such primes is $\ll n^{2 / 3}$, thus

$$
\sum_{n^{2 / 3} \leq p \leq 2 n}\left(\beta_{p}(n)-\beta_{p}^{*}(n)-\alpha_{p}(n)+\alpha_{p}^{*}(n)\right) \log p \ll n^{2 / 3} \log n .
$$

We continue estimating the second sum in Equation (6):

Lemma 4.3. The following estimate holds:

$$
\sum_{n^{2 / 3} \leq p \leq 2 n} \beta_{p}^{*}(n) \log p=n+O\left(\frac{n}{\log n}\right) .
$$

Proof. Summing by parts and using estimate (4) for $\pi_{1}(x)$ we get:

$$
\begin{aligned}
\sum_{n^{2 / 3} \leq p \leq 2 n} \beta_{p}^{*}(n) \log p & =\sum_{\substack{n^{2 / 3} \leq p \leq 2 n \\
p \equiv 1(\bmod 4)}} \log p \\
& =\sum_{\substack{p \leq 2 n \\
p \equiv 1(\bmod 4)}} \log p+O\left(n^{2 / 3}\right) \\
& =\log (2 n) \pi_{1}(2 n)-\int_{2}^{2 n} \frac{\pi_{1}(t)}{t} \mathrm{~d} t+O\left(n^{2 / 3}\right) \\
& =n+O\left(\frac{n}{\log n}\right) .
\end{aligned}
$$

Finally, we deal with the contribution of the coefficients $\alpha_{p}^{*}$. In this point we need to take care of the error term in a more detailed way:

Lemma 4.4. For any $\delta<8 / 9$ the following estimate holds:

$$
\sum_{n^{2 / 3} \leq p \leq 2 n} \alpha_{p}^{*}(n) \log p=\sum_{n^{2 / 3} \leq p \leq 2 n} \frac{\left(1+\left(\frac{-1}{p}\right)\right) n \log p}{p-1}+O\left(\frac{n}{(\log n)^{\delta / 2}}\right) .
$$


Proof. Using (5) and noting that $\nu_{1}+\nu_{2}=p$, where $1 \leq \nu_{1}, \nu_{2} \leq p$ are solutions of $i^{2} \equiv$ $-1(\bmod p)$, we get

$$
\begin{aligned}
\alpha_{p}^{*}(n) & =2+\left\lfloor\frac{n-\nu_{1}}{p}\right\rfloor+\left\lfloor\frac{n-\nu_{2}}{p}\right\rfloor \\
& =2+\frac{2 n}{p}-\frac{\nu_{1}+\nu_{2}}{p}-\left\{\frac{n-\nu_{1}}{p}\right\}-\left\{\frac{n-\nu_{2}}{p}\right\} \\
& =\frac{2 n}{p}+\frac{1}{2}-\left\{\frac{n-\nu_{1}}{p}\right\}+\frac{1}{2}-\left\{\frac{n-\nu_{2}}{p}\right\}
\end{aligned}
$$

so the sum over all primes in the interval $\left[n^{2 / 3}, 2 n\right]$ is equal to

$$
\sum_{n^{2 / 3} \leq p \leq 2 n} \alpha_{p}^{*}(n) \log p=\sum_{n^{2 / 3} \leq p \leq 2 n} \frac{\left(1+\left(\frac{-1}{p}\right)\right) n \log p}{p}+\sum_{\substack{n^{2 / 3} \leq p \leq 2 n \\ \nu^{2} \equiv-1(\bmod p) \\ 0 \leq \nu<p}} \log p\left(\frac{1}{2}-\left\{\frac{n-\nu}{p}\right\}\right) .
$$

We rewrite

$$
\sum_{n^{2 / 3} \leq p \leq 2 n} \frac{\left(1+\left(\frac{-1}{p}\right)\right) n \log p}{p}=\sum_{n^{2 / 3} \leq p \leq 2 n} \frac{\left(1+\left(\frac{-1}{p}\right)\right) n \log p}{p-1}+O\left(n^{1 / 3} \log n\right)
$$

and

$$
\sum_{\substack{n^{2 / 3} \leq p \leq 2 n \\ \nu^{2} \equiv-1(\bmod p) \\ 0 \leq \nu<p}} \log p\left(\frac{1}{2}-\left\{\frac{n-\nu}{p}\right\}\right)=\log n \sum_{\substack{0<\nu<p \leq 2 n \\ \nu^{2} \equiv-1(\bmod p)}}\left(\frac{1}{2}-\left\{\frac{n-\nu}{p}\right\}\right)+O\left(\frac{n}{\log n}\right) .
$$

Notice that for any sequence $a_{p}$ satisfying $a_{p} \ll 1$ we have by a summing by parts argument that

$$
\sum_{p<x} a_{p} \log p=\log x \sum_{p<x} a_{p}-\int_{1}^{x} \frac{1}{t} \sum_{p<t} a_{p} \mathrm{~d} t=\log x \sum_{p<x} a_{p}+O\left(\frac{x}{\log x}\right) .
$$

In order to get the claimed bound, it remains to show that

$$
\sum_{\substack{0<\nu<p \leq 2 n \\ \nu^{2} \equiv-1(\bmod p)}}\left(\frac{1}{2}-\left\{\frac{n-\nu}{p}\right\}\right)=O\left(\frac{n}{(\log n)^{1+\delta / 2}}\right) .
$$

To do that, we divide the summation interval into $1+H$ parts $[1,2 n]=[1, A] \cup L_{1} \cup \cdots \cup L_{H}$, where

$$
L_{i}=\left(\frac{2 n A H}{2 n(H-i+1)+A(i-1)}, \frac{2 n A H}{2 n(H-i)+A i}\right] .
$$

We choose $A=\left\lfloor n /(\log n)^{\delta / 2}\right\rfloor$ and $H=\left\lfloor(\log n)^{\delta}\right\rfloor$ in order to minimize the error term, but we continue using these notations for the sake of conciseness.

Observe that in every of these parts, except the first one, $n / p$ is almost constant, which enables to use the fact that $\nu / p$ is well distributed. More precisely, if $p \in L_{i}$ then

$$
\frac{n}{p} \in\left[\lambda_{i}, \lambda_{i-1}\right):=\left[\frac{2 n(H-i)+A i}{2 A H}, \frac{2 n(H-i+1)+A(i-1)}{2 A H}\right),
$$

and the length of such interval is small: $\left|\left[\lambda_{i}, \lambda_{i-1}\right)\right|=\frac{2 n-A}{2 A H}$. We would then like to replace $\frac{n}{p}$ by $\lambda_{i}$ whenever $\frac{n}{p} \in\left[\lambda_{i}, \lambda_{i-1}\right)$ using

$$
\left\{\frac{n}{p}-\frac{\nu}{p}\right\}=\left\{\lambda_{i}-\frac{\nu}{p}\right\}+\left\{\frac{n}{p}-\lambda_{i}\right\},
$$


but this equality does not hold if $\lambda_{i}<\frac{\nu}{p}+k<\frac{n}{p}$ for some integer $k$. We extend this interval to $\lambda_{i} \leq \frac{\nu}{p}+k \leq \lambda_{i-1}$, rewrite it as $\frac{\nu}{p} \in\left[\lambda_{i}, \lambda_{i-1}\right]_{1}$ and split the previous sum into three parts:

$$
\sum_{\substack{0<\nu<p \leq 2 n \\ \nu^{2} \equiv-1(\bmod p)}}\left(\frac{1}{2}-\left\{\frac{n-\nu}{p}\right\}\right)=\Sigma_{1}+\Sigma_{2}+\Sigma_{3}+O\left(\pi_{1}(A)\right),
$$

where $\Sigma_{1}, \Sigma_{2}$ and $\Sigma_{3}$ are defined as

$$
\begin{aligned}
& \Sigma_{1}=\sum_{i=1}^{H} \sum_{\substack{0 \leq \nu<p \in L_{i} \\
\nu^{2} \equiv-1(\bmod p)}}\left(\frac{1}{2}-\left\{\lambda_{i}-\frac{\nu}{p}\right\}\right), \\
& \Sigma_{2}=\sum_{i=1}^{H} \sum_{\substack{0 \leq \nu<p \in L_{i} \\
\nu^{2} \equiv-1(\bmod p) \\
\frac{\nu}{p} \notin\left[\lambda_{i}, \lambda_{i-1}\right]_{1}}}\left(\left\{\lambda_{i}-\frac{\nu}{p}\right\}-\left\{\frac{n}{p}-\frac{\nu}{p}\right\}\right), \\
& \Sigma_{3}=\sum_{i=1}^{H} \sum_{\substack{0 \leq \nu<p \in L_{i} \\
\nu^{2} \equiv-1(\bmod p) \\
\frac{\nu}{p} \in\left[\lambda_{i}, \lambda_{i-1}\right]_{1}}}\left(\left\{\lambda_{i}-\frac{\nu}{p}\right\}-\left\{\frac{n}{p}-\frac{\nu}{p}\right\}\right) .
\end{aligned}
$$

Recall that $A=n /(\log n)^{\delta / 2}+O(1)$ and $H=(\log n)^{\delta}+O(1)$, so $\pi_{1}(A)=O\left(n /(\log n)^{1+\delta / 2}\right)$. We now estimate each of the sums $\Sigma_{1}, \Sigma_{2}, \Sigma_{3}$ separately, making use of Lemma 2.1. For the first one note that

$$
\int_{0}^{1}\left(\frac{1}{2}-\left\{\lambda_{i}-t\right\}\right) \mathrm{d} t=0
$$

so we get

$$
\begin{aligned}
\Sigma_{1} & =\sum_{i=1}^{H} \sum_{\substack{0 \leq \nu<p \in L_{i} \\
\nu^{2} \equiv-1(\bmod p)}}\left(\frac{1}{2}-\left\{\lambda_{i}-\frac{\nu}{p}\right\}\right) \\
& =\sum_{i=1}^{H} O\left(\frac{2 n A H}{2 n(H-i)+A i} /\left(\log \frac{2 n A H}{2 n(H-i)+A i}\right)^{1+\delta}\right) \\
& =O\left(\frac{2 n A H}{(\log n)^{1+\delta}} \int_{0}^{H} \frac{\mathrm{d} i}{2 n(H-i)+A i}\right) \\
& =O\left(\frac{2 n A H}{(\log n)^{1+\delta}} \frac{\log 2 n / A}{2 n-A}\right)=O\left(\frac{n \log \log n}{(\log n)^{1+\delta / 2}}\right) .
\end{aligned}
$$

For the second sum we use Equation (17):

$$
\begin{aligned}
\Sigma_{2} & =\sum_{i=1}^{H} \sum_{\substack{0 \leq \nu<p \in L_{i} \\
\nu^{2} \equiv-1(\bmod p) \\
\frac{\equiv}{p} \notin\left[\lambda_{i}, \lambda_{i-1}\right]_{1}}}\left\{\frac{n}{p}-\lambda_{i}\right\} \\
& \leq \sum_{i=1}^{H} \sum_{\substack{0 \leq \nu<p \in L_{i} \\
\nu^{2} \equiv-1(\bmod p)}}\left|\left[\lambda_{i}, \lambda_{i-1}\right]\right| \\
& \leq \frac{2 n-A}{2 A H} 2 \pi_{1}(2 n)=O\left(\frac{n}{(\log n)^{1+\delta / 2}}\right) .
\end{aligned}
$$


Finally, for the third sum we use the notation $\mathbb{I}_{\left[\lambda_{i}, \lambda_{i-1}\right]_{1}}$ for the indicator function of the interval $\left[\lambda_{i}, \lambda_{i-1}\right]$ modulo 1 , which satisfies

$$
\int_{0}^{1} \mathbb{I}_{\left[\lambda_{i}, \lambda_{i-1}\right]_{1}}(t) \mathrm{d} t=\left|\left[\lambda_{i}, \lambda_{i-1}\right]\right|
$$

so using Lemma 2.1 we get

$$
\begin{aligned}
\Sigma_{3} & \ll \sum_{i=1}^{H} \sum_{\substack{0 \leq \nu<p \in L_{i} \\
\nu^{2} \equiv-1(\bmod p) \\
\frac{\nu}{p} \in\left[\lambda_{i}, \lambda_{i-1}\right]_{1}}} 1=\sum_{i=1}^{H} \sum_{\substack{0 \leq \nu<p \in L_{i} \\
\nu^{2} \equiv-1(\bmod p)}} \mathbb{I}_{\left[\lambda_{i}, \lambda_{i-1}\right]_{1}}\left(\frac{\nu}{p}\right) \\
& =\sum_{i=1}^{H} 2 \pi_{i}\left(L_{i}\right)\left|\left[\lambda_{i}, \lambda_{i-1}\right]\right|+O\left(\frac{2 n A H}{2 n(H-i)+A i} /\left(\log \frac{2 n A H}{2 n(H-i)+A i}\right)^{1+\delta}\right) . \\
& =O\left(\frac{n \log \log n}{(\log n)^{1+\delta / 2}}\right) . \quad(\text { continuing as from }(*) \text { and }(* *))
\end{aligned}
$$

Finally, we note that any function $f$ satisfying $f(n)=O\left(\frac{n \log \log n}{(\log n)^{1+\delta / 2}}\right)$ for every $\delta<8 / 9$ also satisfies $f(n)=O\left(\frac{n}{(\log n)^{1+\delta / 2}}\right)$ for every $\delta<8 / 9$, hence this concludes the proof.

\section{Proof of theorem 1.1}

Combining results from Lemmas 3.3, 4.1, 4.3 and 4.4 and taking $\theta=\delta / 2$, we get that for any constant $\theta<4 / 9$

$$
\log L_{n}=2 n \log n-n\left(1+\frac{\log 2}{2}+\sum_{2<p \leq 2 n} \frac{\left(1+\left(\frac{-1}{p}\right)\right) \log p}{p-1}\right)+O\left(\frac{n}{(\log n)^{\theta}}\right) .
$$

Note that,

$$
\sum_{2<p \leq 2 n} \frac{\left(1+\left(\frac{-1}{p}\right)\right) \log p}{p-1}=\sum_{2<p \leq 2 n} \frac{\log p}{p-1}+\sum_{2<p \leq 2 n} \frac{\left(\frac{-1}{p}\right) \log p}{p-1},
$$

where the second sum is bounded, since the sum over all primes is convergent. We finish the proof by estimating both sums in two following lemmas:

Lemma 5.1. The following estimate holds:

$$
\sum_{2<p \leq 2 n} \frac{\log p}{p-1}=\log n-\gamma+O\left(\frac{1}{\log n}\right) .
$$

Proof. This estimate is well-known. However, for completeness, we include a detailed proof. Write

$$
\begin{aligned}
\sum_{p \leq x} \frac{\log p}{p\left(1-\frac{1}{p}\right)} & =\sum_{p \leq x} \frac{\log p}{p}\left(1+\frac{1}{p}+\frac{1}{p^{2}}+\cdots\right) \\
& =\sum_{p^{j} \leq x} \frac{\log p}{p^{j}}+\sum_{\substack{p^{j}>x \\
p \leq x}} \frac{\log p}{p^{j}} .
\end{aligned}
$$

By Merten's theorem

$$
\sum_{p^{j} \leq x} \frac{\log p}{p^{j}}=\log x-\gamma+o(1)
$$


and the error term can be improved using Prime Number Theorem in the form (3) and summation by parts:

$$
\begin{aligned}
\sum_{p^{j} \leq x} \frac{\log p}{p^{j}} & =\frac{\psi(x)}{x}+\int_{2}^{x} \frac{\psi(t)}{t^{2}} \mathrm{~d} t \\
& =1+O\left(\frac{1}{\log x}\right)+\int_{2}^{x} \frac{1}{t} d t+\int_{2}^{x} \frac{E(t)}{t^{2}} d t \\
& =1+O\left(\frac{1}{\log x}\right)+\log x-\log 2+\int_{2}^{\infty} \frac{E(t)}{t^{2}} d t-\int_{x}^{\infty} \frac{E(t)}{t^{2}} d t \\
& =\log x-\gamma+O\left(\frac{1}{\log x}\right) .
\end{aligned}
$$

For the second term we have

$$
\begin{aligned}
\sum_{\substack{p^{j}>x \\
p \leq x}} \frac{\log p}{p^{j}} & =\sum_{p \leq x} \frac{\log p}{\left.p^{\lfloor\log x}\right\rfloor \cdot p}\left(1+\frac{1}{p}+\frac{1}{p^{2}}+\cdots\right) \\
& \leq \frac{1}{x^{1 / 2}} \sum_{p \leq x} \frac{\log p}{p\left(1-\frac{1}{p}\right)} \\
& =O\left(\frac{\log x}{x^{1 / 2}}\right) .
\end{aligned}
$$

In our case we get

$$
\sum_{2<p \leq 2 n} \frac{\log p}{p-1}=\sum_{p \leq 2 n} \frac{\log p}{p-1}-\log 2=\log n-\gamma+O\left(\frac{1}{\log n}\right) .
$$

Remark: The error term in the previous lemma can be sharpened to $O(\exp (-c \sqrt{\log n}))$ (where $c$ is a constant) using the estimate

$$
\sum_{p^{j} \leq x} \frac{\log p}{p^{j}}=\log x-\gamma+O(\exp (-c \sqrt{\log x}))
$$

which can be found, for instance, in [6] (Exercise 4, page 182).

Lemma 5.2. The following estimate holds:

$$
\sum_{2<p \leq 2 n} \frac{\left(\frac{-1}{p}\right) \log p}{p-1}=\sum_{p \neq 2} \frac{\left(\frac{-1}{p}\right) \log p}{p-1}+O\left(\frac{1}{\log n}\right) .
$$

Proof. We know that

$$
\sum_{2<p \leq 2 n} \frac{\left(\frac{-1}{p}\right) \log p}{p-1}=\sum_{p \neq 2} \frac{\left(\frac{-1}{p}\right) \log p}{p-1}+o(1),
$$

since the sum over all primes is convergent. Alternatively, we can express the sum as follows

$$
\sum_{2<p \leq 2 n} \frac{\left(\frac{-1}{p}\right) \log p}{p-1}=\sum_{\substack{2<p \leq 2 n \\ p \equiv 1 \bmod 4}} \frac{\log p}{p-1}-\sum_{\substack{2<p \leq 2 n \\ p \equiv 3 \bmod 4}} \frac{\log p}{p-1} .
$$

It follows from Prime Number Theorem in arithmetic progressions that

$$
\psi_{1}(x):=\sum_{\substack{p^{j}<x \\ p \equiv 1 \bmod 4}} \log p=\frac{x}{2}+E_{1}(x), E_{1}(x)=O\left(\frac{x}{(\log x)^{2}}\right) .
$$


(equivalently for $\psi_{3}(x)$, where the summation is over primes equivalent to 3 modulo 4 ). We can use this as in proof of Lemma 5.1 to get

$$
\begin{gathered}
\sum_{\substack{2<p \leq 2 n \\
p \equiv 1 \bmod 4}} \frac{\log p}{p-1}=\frac{1}{2} \log n+C_{1}+O\left(\frac{1}{\log n}\right), \\
\sum_{\substack{2<p \leq 2 n \\
p \equiv 3 \bmod 4}} \frac{\log p}{p-1}=\frac{1}{2} \log n+C_{3}+O\left(\frac{1}{\log n}\right) .
\end{gathered}
$$

The difference $C_{1}-C_{3}$ then has to be equal to $\sum_{p \neq 2} \frac{\left(\frac{-1}{p}\right) \log p}{p-1}$ and we get the required convergence rate.

Acknowledgments: This work was done during second author's visit at Universidad Autónoma de Madrid in Winter of 2011. He would like to thank people of Mathematics Department and especially Javier Cilleruelo for their warm hospitality. The authors are also grateful for his advice and helpful suggestions in the preparation of this paper.

Pieter Moree is greatly thanked for noticing the connection between the constant (2) and the non-hypotenuse numbers, for pointing reference [6] in relation to Lemma 5.1 and also for useful comments.

The first author is supported by a JAE-DOC grant from the JAE program in CSIC, Spain. The last author is supported by Departamento de Matemáticas of Universidad Autónoma de Madrid, Spain.

\section{REFERENCES}

[1] P. Bateman, J. Kalb, and A. Stenger. A limit involving least common multiples. American Mathematical Monthly, 109:393-394, 2002.

[2] J. Cilleruelo. The least common multiple of a quadratic sequence. Compositio Mathematica, 147(4):1129-1150, 2011.

[3] W. Duke, J. Friedlander, and H. Iwaniec. Equidistribution of roots of a quadratic congruence to prime moduli. Annals of Mathematics, 141(2):423-441, 1995.

[4] K. Homma. On the discrepancy of uniformly distributed roots of quadratic congruences. Journal of Number Theory, 128(3):500-508, 2008.

[5] S. Hong, G. Quian, and Q. Tan. The least common multiple of sequence of product of linear polynomials. Acta Mathematica Hungarica (to appear).

[6] H. L. Montgomery and R. C. Vaughan. Multiplicative number theory. I. Classical theory, volume 97 of Cambridge Studies in Advanced Mathematics. Cambridge University Press, Cambridge, 2007.

[7] P. Moree. Counting numbers in multiplicative sets: Landau versus Ramanujan. preprint, available on-line at arXiv:1110.0708v1, 2011.

[8] H. Niederreiter. Random number generation and quasi-Monte Carlo methods, volume 63 of CBMS-NSF Regional Conference Series in Applied Mathematics. Society for Industrial and Applied Mathematics (SIAM), 1992.

[9] Á. Tóth. Roots of quadratic congruences. Internat. Math. Res. Notices, (14):719-739, 2000.

J. Rué: Instituto de Ciencias Matemáticas (CSIC-UAM-UC3M-UCM), 28049 Madrid, Spain

E-mail address: juanjo.rue@icmat.es

P. Šarka: Institute of Mathematics and Informatics, Akademijos 4, Vilnius LT-08663, Lithuania and Department of Mathematics and Informatics, Vilnius University, Naugarduko 24, Vilnius LT-03225, LiTHUANIA

E-mail address: paulius.sarka@gmail.com

A. Zumalacárregui: Instituto de Ciencias Matemáticas (CSIC-UAM-UC3M-UCM) and Departamento de Matemáticas, Universidad Autónoma de Madrid, 28049 Madrid, Spain

E-mail address: ana.zumalacarregui@uam.es 